\title{
Clinical manifestations in HTLV-1 infected individuals without HAM/TSP: Results of an 8 years cohort
}

\author{
Davi Tanajura*, Néviton Castro, Paulo Oliveira, Glória Orge, Anselmo Souza, Natália Barbosa, Silvane Santos, \\ Edgar Carvalho
}

From 16th International Conference on Human Retroviruses: HTLV and Related Viruses Montreal, Canada. 26-30 June 2013

\section{Introduction}

HTLV-1 is an endemic virus in the northeast of Brazil and is related to HAM/TSP in up to $5 \%$ of infected individuals. Despite this low prevalence it is known that a higher number of carriers presents with clinical and neurological symptoms that can be sensory, motor, urinary, inflammatory or autonomic.

\section{Method}

This is a cohort study with 440 HTLV-1 infected individuals in Salvador, Bahia. Symptoms were computed every year and incidence rates were calculated to each variable in data.

\section{Results}

Applying international criteria 163 individuals had definite, possible or probable HAM/TSP and 266 were asymptomatic. The last was selected for analysis. Followup of at least one year was archived in 64 to $75 \%$ of patients, varying according to the studied variable. The symptoms incidence rate was $20.6 \%$ for hand numbness, $18.6 \%$ for feet numbness, $12.9 \%$ for nocturia, $12.6 \%$ for urgency and $13.3 \%$ for dry mouth. In the physical exam the incidence was $22.7 \%$ for gingivitis, $7.6 \%$ for legs hyperreflexia, $5.2 \%$ for legs paraparesis and $3.6 \%$ for Babinski sign. In annual applied EDSS scale the incidence rate of worsening 1 point was $13.4 \%$. Cox regression was done with EDSS curves and identified proviral load and female gender as significant risk factors (ORs 3.36 [1-11.66] and 1.80 [1.09-2.97] respectively). Six patients developed HAM/TSP during the cohort.

\footnotetext{
* Correspondence: davicostamd@gmail.com

Immunology Department, Universidade Federal da Bahia (UFBA), Salvador,
} Bahia, Brazil 